\title{
CRASHWORTHINESS AND COMPARATIVE ANALYSIS OF POLYGONAL SINGLE AND BI-TUBULAR STRUCTURES UNDER AXIAL LOADING - EXPERIMENTS AND FE MODELLING
}

\author{
I. VIMAL KANNAN \\ SSM Institute of Engineering and Technology, Faculty of Mechanical Engineering, Dindigul, India \\ e-mail: ivimalkannan@gmail.com \\ R. RAJKUMAR \\ Mepco Schlenk Engineering College, Faculty of Mechanical Engineering, Sivakasi, India \\ e-mail: rrkumarau@gmail.com
}

\begin{abstract}
This article aims to present a report of experimental and numerical investigations on crashworthiness characteristics of single and multi-cell/bi-tubular structures. Novel multi-cell/bi-tubular structures are proposed in order to improve the crashworthiness performance, LS-DYNA FE software is applied for the modelling of axial crashing behaviour to validate with experimental results and a good agreement is observed. The KPIs are used to compare various structures and to determine the best performing ones. The investigations reveal that the HMC4 has significantly obvious effects on the structural crashworthiness and improved 515\% energy absorption efficiency. Afterward, a parametric study has been carried out for the best energy absorber.
\end{abstract}

Keywords: crashworthiness, thin-walled structures, single and multi-cell tubular structures, energy absorption, key performance indicators

\section{Introduction}

Thin-walled tubes, particularly metal tubular structures, were used as impact energy absorbers in all types of transport systems such as automobile, aircraft, military and defense equipment owing to their desirable properties like lightweight, easy formation and more energy absorption capability. Professionals and researchers have conducted a large number of effective researches on energy absorption performance of thin-walled structures through experimental works, theoretical and numerical simulations (Baroutaji et al., 2017). For example, Olabi et al. (2007) constructed a nested structure in thin-walled cylinders, which improved total energy absorption and the mean crashing force. With the intention of further enhancement of the energy absorption capability, fillers like foam or honeycomb were filled in a tubular structure, and it was found that a significant improvement in energy absorption by the effect of interaction between filler materials and tube wall (Hussein et al., 2017; Mozafari et al., 2016) have been carried out extensively.

Multi-cellular thin-walled structures are a one type of energy absorbing-structures. Some researchers studied the effect of geometry and the number of cells of multi-cellular structures on crashworthiness (Yin et al., 2014; Sun et al., 2018). Their findings showed that specific energy absorption and the mean crashing force of multi-cellular tubes escalated with an increase in the number of cells (Zhang et al., 2018a; Wu et al., 2016).

In recent years, the crashing behaviour of bi-tubes with different geometric sections was investigated by Nia and Parsapour (2014), Sharifi et al. (2015), Vinayagar and Kumar (2017a). They found that the bi-tubes were more efficient compared to simple tubes in energy absorption. 
Many scholars optimized the crashworthiness parameters of multi-cell/bi-tubular thin-walled tubes (Sun et al., 2017; Pyrz and Krzywoblocki, 2017; Vinayagar and Kumar, 2017b; Bigdeli and Nouri, 2019; Deng and Liu, 2019).

In addition to that, the combined effects of structural dimension on cost and performance were analyzed by Sun et al. (2019). Their proposed design methodology allowed them to better balance the performance and costs in mass production. Some of the researchers (Yu et al., 2014; Umeda et al., 2010; Abramowicz and Jones, 1984, 1986; Markiewicz et al., 1998; Li et al., 2018; Zhang et al., 2018b) proposed a set of Key Performance Indicators (KPIs) to predict, evaluate and compare the energy absorption performance of tubular sections with various configurations. Key Performance Indicators (KPI) were used to evaluate the energy absorbing capacity of polygonal single and bi-tubular tubes. They concluded that the hexagonal bi-tubes have more energy absorption capacity than the other single and bi-tubular sections.

Inspired with a large number of previous surveys, four types of multi-cell/bi-tubular structures were proposed. For type 1, multi-cell geometry was taken from Nia and Parsapour (2014), and all other (type 2, 3 and 4) geometries were new. Type 2 multi-cell structure consists of bi-tubes with the same geometry. In type 3, polygonal tubes consist of circular cylinders in a circumscribed manner and in type 4, polygonal tubes consist of circular cylinders in an inscribed manner. In this analysis, nearly 12 configurations of multi-cell structures with different sections were used. The present work is to study and analyse the impact energy absorption of stainless steel thin-walled tubes with respect to their structures. These structures/absorbers offer more chances to identify the best one which increases the energy absorption capability and improves crashworthiness performance.

\section{Experimental procedure}

\subsection{Specimen preparation}

Specimen structures with suitable dimensions were not commercially available. Hence, the specimens were made in a workshop from $1.5 \mathrm{~mm}$ thick ASTM A240 type 304 stainless steel sheets. Stainless steel 304 is considered for its good formability and strength. From the tensile test results, the mechanical properties (ASTM A 370-2017) of stainless steel 304 sheet materials are assessed by utilizing the relations and were classified in Table 1.

Table 1. Mechanical properties of the materials

\begin{tabular}{|l|c|}
\hline \multicolumn{1}{|c|}{ Property } & Value \\
\hline \hline Tensile strength [MPa] & 883.02 \\
\hline Yield stress [MPa] & 617.08 \\
\hline Percent of elongation at break $e_{b}[\%]$ & 46.56 \\
\hline Poisson's ratio $\nu[-]$ & 0.3 \\
\hline Density $\rho\left[\mathrm{N} / \mathrm{m}^{3}\right]$ & 8000 \\
\hline Young's modulus $E[\mathrm{MPa}]$ & 207.8 \\
\hline
\end{tabular}

All the specimens have been prepared with the same height, average sectional area and volume. The side of a polygonal section was derived from the inscribed diameter of the polygonal sections and had the diameter of $52 \mathrm{~mm}$. For easy identification and evaluation, all the five unique kinds of samples were coded. The first code letter represents the shape, $\mathrm{T}$ for triangular, $\mathrm{S}$ for square, $\mathrm{P}$ for pentagon and $\mathrm{H}$ for Hexagon. The second and third letter represents the simple cell (SC) or multi-cell (MC) structure and the last number represents the kind of multi-cell 
design. For instance, SMC1 implies the first letter represents a square, MC represents multi-cell structure and 1 represents type 1 pattern, see Fig. 1.

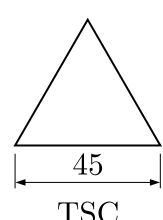

TSC

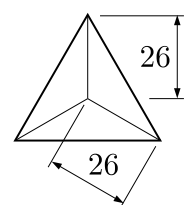

TMC1

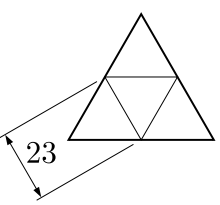

TMC2

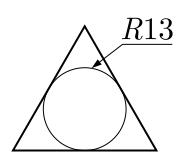

TMC3

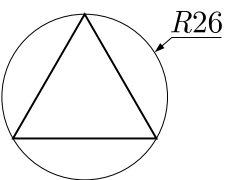

TMC4
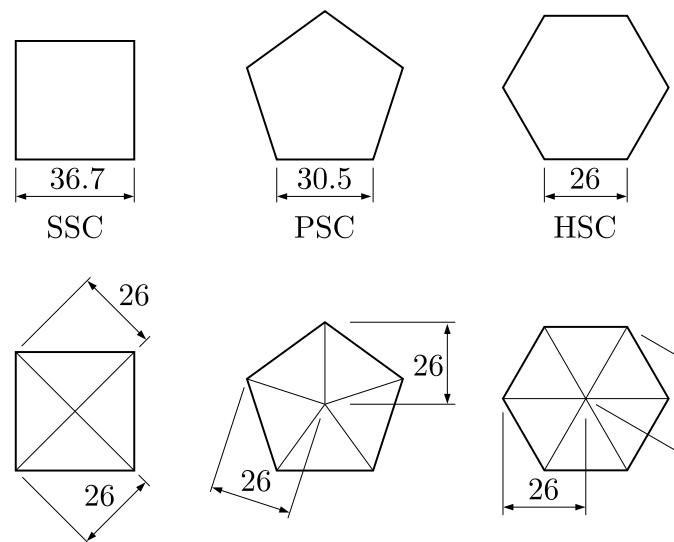

SMC1

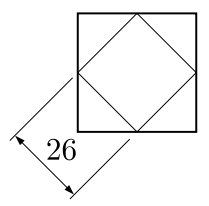

$\mathrm{SMC} 2$

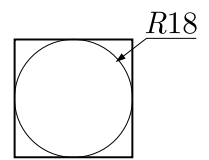

SMC3

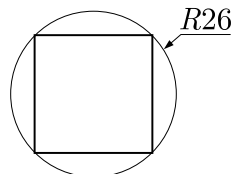

SMC4

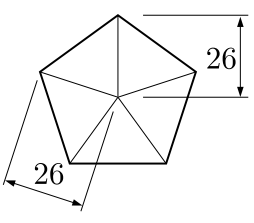

PMC1

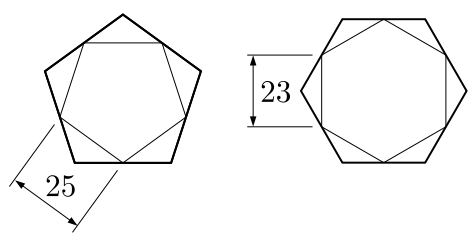

HMC2

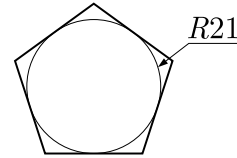

PMC3

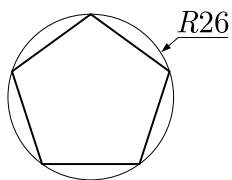

PMC4

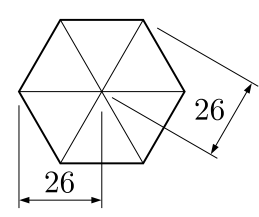

HMC1

Fig. 1. Designed pattern

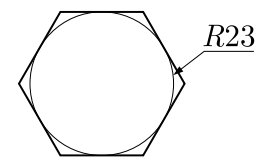

HMC3

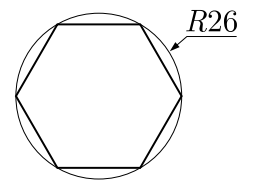

HMC4

\subsection{Experimental process}

In the analysis, the fabricated specimens were subjected to a quasi-static axial crashing test in the servo-controlled universal testing machine of FSA makes, Model TUF-CN 1000 at a loading rate of $10 \mathrm{~mm} / \mathrm{min}$. That UTM has two jaws, the lower one was movable and the upper one was stationary, the specimens were placed between them. No fixture was used in the axial compression; faceplates were placed in the upper and lower part of the samples. Concentric circles were drawn in the lower part of the table and the sample was placed and coincided with the circles.

The specimens were crashed up to $80 \mathrm{~mm}$ at the room temperature of $30^{\circ} \mathrm{C}$. The test was repeated for 3 times and the best results were taken into account. The load-displacement curve for the test is represented in Fig. 2. Figure 3 shows some of the specimens before, during and after loading. 
(a)
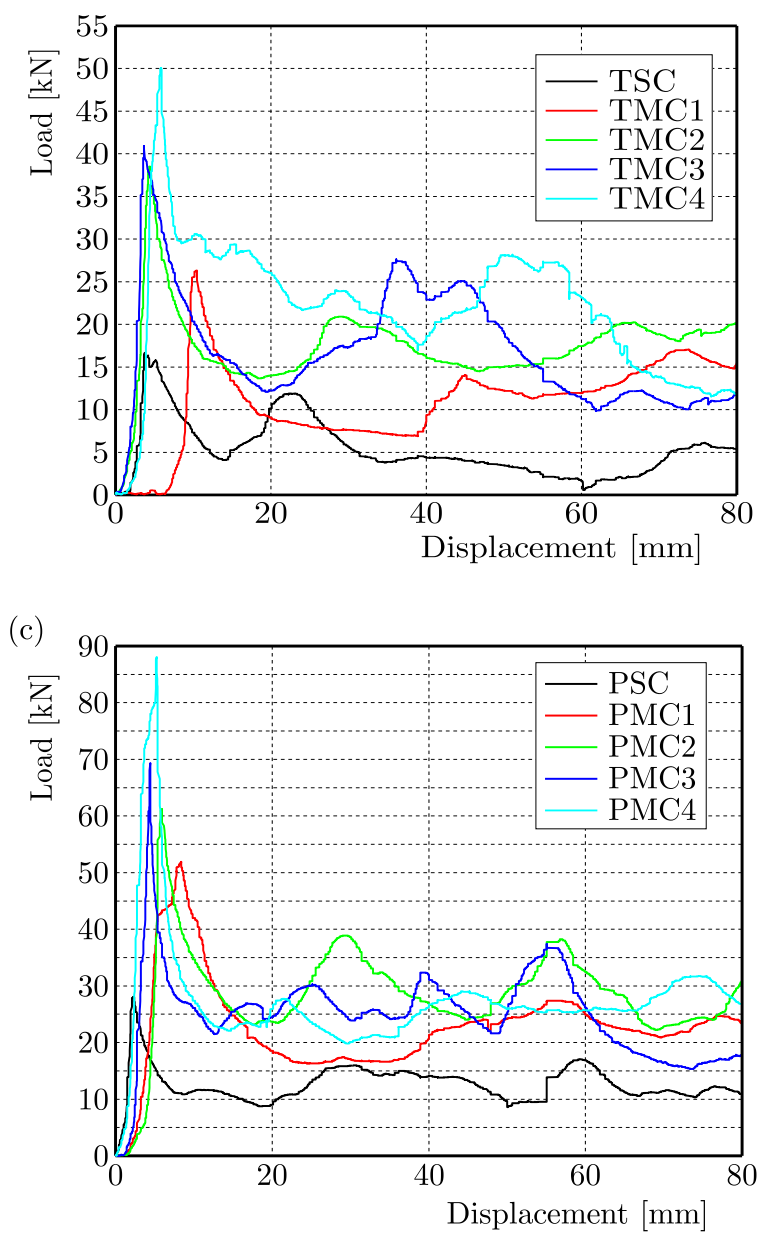

(b)

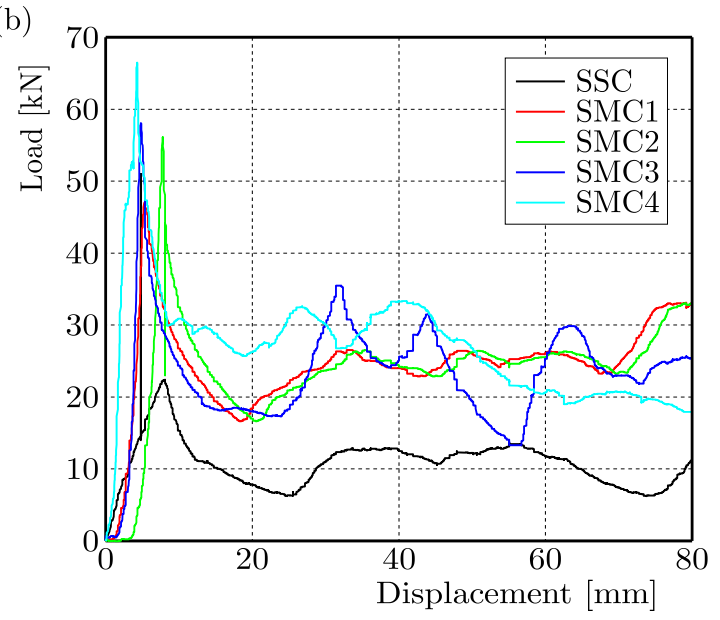

(d)

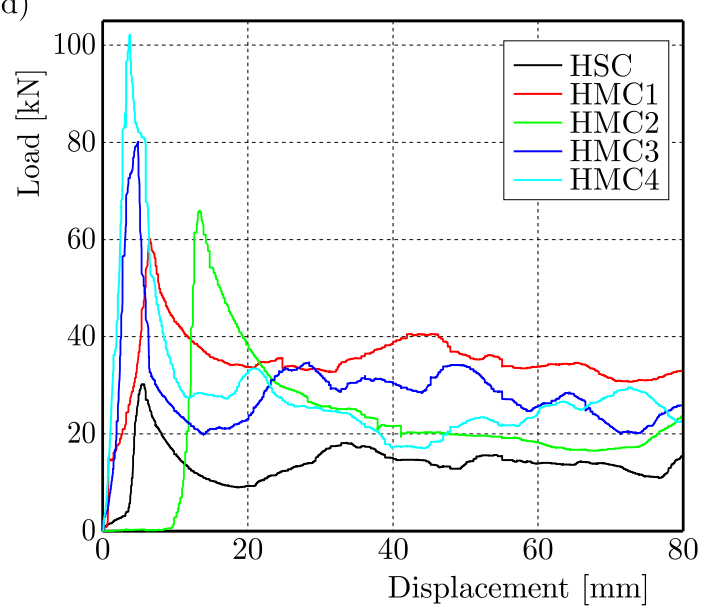

Fig. 2. Load-displacement curves of experiments: (a) triangular tubes, (b) square tubes, (c) pentagonal tubes and (d) hexagonal tubes
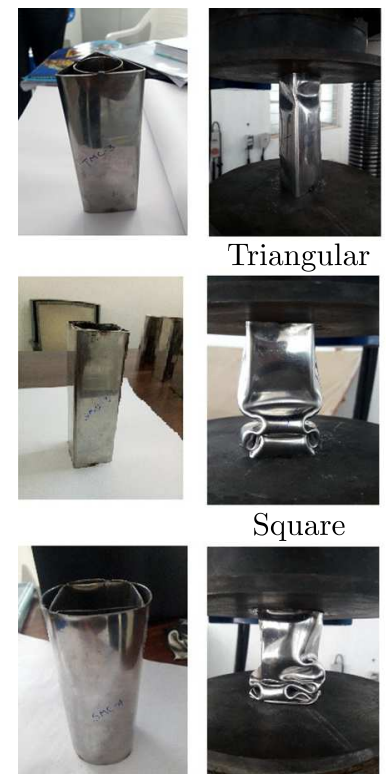

Square

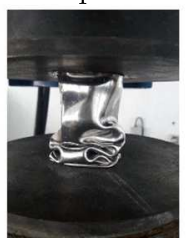

Circular
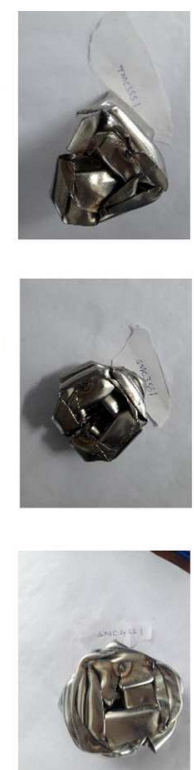
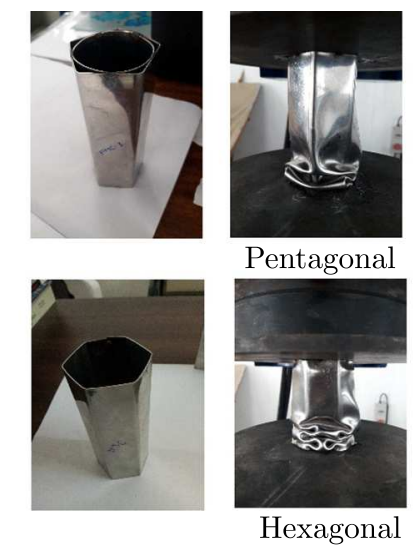
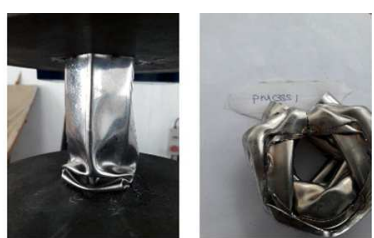

Pentagonal

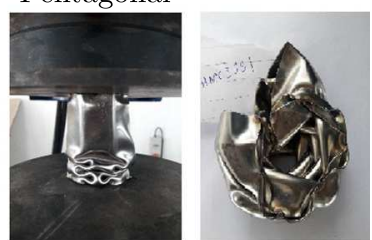

Hexagonal

Fig. 3. Sample of specimens before, during and after loading 


\section{Key performance indicators}

The Key Performance Indicators (KPIs) (Yu et al., 2014) were important five factors like Effective Stroke Ratio (ESR), Specific Energy Absorption (SEA), Effectiveness of Energy Absorption (EEA), Non-dimensional Load-carrying Capacity (NLC), Stability of Load-carrying Capacity (SLC) depicted underneath with numerical formulas.

\subsection{Effective Stroke Ratio (ESR)}

ESR is defined as the ratio of the effective stroke $\delta_{\text {ef }}$ to the total length of the tube under compression. It is a non-dimensional indicator characterising the effective utilization rate of the material being used in the energy absorber. For cellular materials, the concept of ESR is used under and similar to the densification strain

$$
\mathrm{ESR}=\frac{\delta_{e f}}{L}
$$

\subsection{Specific Energy Absorption (SEA)}

Specific energy absorption is considered as retained energy per unit mass of the thin-walled sections. It is one of the common criteria for comparing the energy absorption capacity of structures with distinctive mass which is given by

$$
\mathrm{SEA}=\frac{\mathrm{TEA}}{m}
$$

where TEA is the Total Energy Absorption, TEA $=\int_{0}^{\delta} F(\delta) d \delta$ and $F$ is the direct crashing force with a work of the crashing distance $\delta ; m$ is mass of the structure.

\subsection{Effectiveness of Energy Absorption (EEA)}

EEA is directly proportional to the mean crashing force, ESR and indirectly proportional to the net cross-sectional area and yield stress of the material. It is a non-dimensional indicator which indicates the volumetric efficiency of the tubes under compression

$$
\mathrm{EEA}=\frac{F_{\text {mean }} \cdot \mathrm{ESR}}{A Y}
$$

where mean crashing force $F_{\text {mean }}=$ TEA $/ \delta$ and TEA is energy absorbed during collapse and crashing distance $\delta ; A$ is apparent area, $Y$ is yield stress.

\subsection{Non-dimensional Load-carrying Capacity (NLC)}

NLC is defined as the ratio between the mean crashing force $F_{\text {mean }}$ and bending moment per unit length $M_{0}$. NLC is a non-dimensional indicator which indicates the effective load-carrying capacity of the specimens

$$
\mathrm{NLC}=\frac{F_{\text {mean }}}{M_{0}}
$$

where $M_{0}$ is the plastic bending moment per unit length $M_{0}=(2 Y / \sqrt{3})\left(t^{2} / 4\right), Y$ is the yield stress of the material and $t$ is thickness of the sample (Yu et al., 2014). 


\subsection{Stableness of Load-carrying Capacity (SLC)}

SLC is defined by the ratio between the mean crashing force and the peak crashing force. It is a non-dimensional indicator which indicates the stability of the load-carrying capacity of the specimens

$$
\mathrm{SLC}=\frac{F_{\text {mean }}}{F_{\text {peak }}}
$$

\subsection{Theoretical predictions}

An analytical solution for the axial compression of thin-walled structures using the super folding element theory has been proposed by many researchers. In this research, investigating and comparing the energy-efficient of thin-walled structures is more important to develop new energy absorbers.

The axial crashing for the resistance of a multi-corner thin-walled tube equal to the crashing force per one element times the number of corner elements. To formulate the axial crashing resistance of thin-walled triangular columns were proposed by Abramowicz and Jones (1986). The mean crashing force of axial crashing resistance can be calculated as

$$
\frac{P_{m}}{M_{0}}=39.17\left(\frac{a}{t}\right)^{0.33}
$$

where the fully plastic bending moment is

$$
M_{0}=\frac{\sigma_{0} t^{2}}{4}
$$

The resistance offered by the average crashing force for the square thin-walled sections was proposed by Abramowicz and Jones (1984) and it can be calculated by

$$
P_{m}=52.22 M_{0} \sqrt[3]{\frac{a}{t}}
$$

The critical expressions of the mean crashing force for hexagonal thin walled sections was reported by Abramowicz and Jones (1986) and it can be calculated by

$$
\frac{P_{m}}{M_{0}}=16.8 \frac{b}{t}+3 \pi \frac{a}{H}+9.56 \frac{H}{b}
$$

where

$$
M_{0}=\sigma_{0} \frac{t^{2}}{4} \quad H=0.821 \sqrt[3]{t a^{2}} \quad b=0.683 \sqrt[3]{t^{2} a}
$$

The refusal to accept the crashing force for circular tubes of thin-walled structures was proposed and expressed by Abramowicz and Jones (1984). The mean crashing force can be calculated by

$$
P_{m}=86.14 M_{0}\left(\frac{a}{t}\right)^{0.33} \quad M_{0}=\frac{2 \sigma_{0}}{\sqrt{3}} \frac{t^{2}}{4}
$$

where $\sigma_{0}$ is the equivalent plastic flow stress, $t$ is the wall thickness, $a$ is the sectional length and $R$ is the mean radius of the circular tube.

The two important structural characteristics indexes of structural effectiveness and solidity ratio have been adopted to estimate the structural performance. The structural effectiveness $\eta$ and solidity ratio $\phi$ are denoted by

$$
\eta=\frac{P_{m}}{A \sigma_{0}} \quad \phi=\frac{A}{A_{0}}
$$


where $A$ and $A_{0}$ denote the net area and apparent area of the tubular cross section. So, the structural effectiveness of the triangular tube $\eta=0.958 \sqrt[3]{\phi^{2}}$, square tube $\eta=2.14 \sqrt[3]{\phi^{2}}$ and circular tube $\eta=2.0 \sqrt[3]{\phi^{2}}$. For thin-walled circular, and polygonal sections we have

$$
\varphi=\frac{4 t}{D}=\frac{4 t}{C}
$$

where $D$ is the diameter of the cylinder and $C$ is the side of the polygon. The theoretical prediction is in good agreement with the experimental results, and this is tabulated in Table 2.

Table 2. Comparison of experimental results with theoretical predictions

\begin{tabular}{|l|c|c|c|c|}
\hline $\begin{array}{c}\text { Specimen } \\
\text { shape }\end{array}$ & $\begin{array}{c}\text { Mean load by } \\
\text { analytical } \\
{[\mathrm{kN}]}\end{array}$ & $\begin{array}{c}\text { Mean load by } \\
\text { experimental } \\
{[\mathrm{kN}]}\end{array}$ & $\begin{array}{c}\text { Differentiate } \\
{[\%]}\end{array}$ & $\begin{array}{c}\text { Structural } \\
\text { effectiveness } \\
\eta\end{array}$ \\
\hline \hline Triangular & 6.80 & 6.33 & 6.97 & 0.26 \\
\hline Square & 9.63 & 10.24 & 6.29 & 0.64 \\
\hline Pentagon & 13.31 & 12.52 & 5.95 & 0.83 \\
\hline Hexagon & 14.90 & 13.69 & 8.14 & 0.85 \\
\hline
\end{tabular}

\section{Results and discussion}

Based on the experimental data of polygonal tubes under axial compression, the stainless steel thin-walled tubes with various cross-sectional patterns including triangular, square, pentagonal, hexagonal and circular ones chosen as evaluation objects. The numerical data are collected from the literature. The influence of cross-section geometry from experimental data is studied, analyzed and compared by utilizing the following KPIs.

In this study, the effective stroke $\delta_{e f}$ is determined from the deformation efficiency $f$ in the following equation

$$
f=\frac{1}{F_{\max }} \int_{0}^{\delta} F \delta d \delta
$$

where $\delta$ is the axial crashing distance, $F$ is the axial crashing force, $F_{\max }$ is the maximum crashing force in the distance 0 to $\delta$. Based on the value of $\delta_{e f}$, the following five KPI's are computed and analyzed.

\subsection{Mode of deformation}

The deformed structures of tested specimens were analyzed and studied. The triangular tubes do not fold gradually; the collapsing procedure begins with the axisymmetric mode and afterward moved to the diamond mode. The length of the fold in the axisymmetric mode is higher than in the diamond mode. The square tubes reveal the symmetric mode of compression, as the applied load folded two faces inward and the opposite two faces folded outwards. The length of fold increased in the subsequent folds after the first fold. The pentagonal tubes collapsed with a complete contraction of all faces, starting with the axisymmetric then symmetric and, finally, diamond mode. The length of the fold increased with the subsequent fold. The symmetric mode of the collapse was observed in hexagonal tubes and ended with the diamond mode. The mixed-mode of compression was resulted by circular tubes; the folding process started with the axisymmetric mode and shifted to the diamond mode. Length of the outer fold of the diamond 
mode was higher than the inward fold of the axisymmetric mode. The load value increased up to the elastic region and dropped down in plastic hinges. Similarly, the number of folds in each specimen was clearly shown in the load-displacement curve. Load-displacement curves obtained from experiments and the calculated energy absorption are tabulated in Table 3.

\subsection{Absorbed energy}

The energy absorption capacity of various specimens is shown in Fig. 4. It is obvious that the hexagon and triangular sections absorbed the most and the least amount of energy between the tested specimens. The pentagonal, square and triangular tubes were set after the hexagonal tube. From the axial compression tests, the larger number of section edges with circumferential tubes had a greater energy absorption capacity. In multi-cell tubes, type 4 was better than type 3 , type 3 than type 2 and type 2 than type 1.

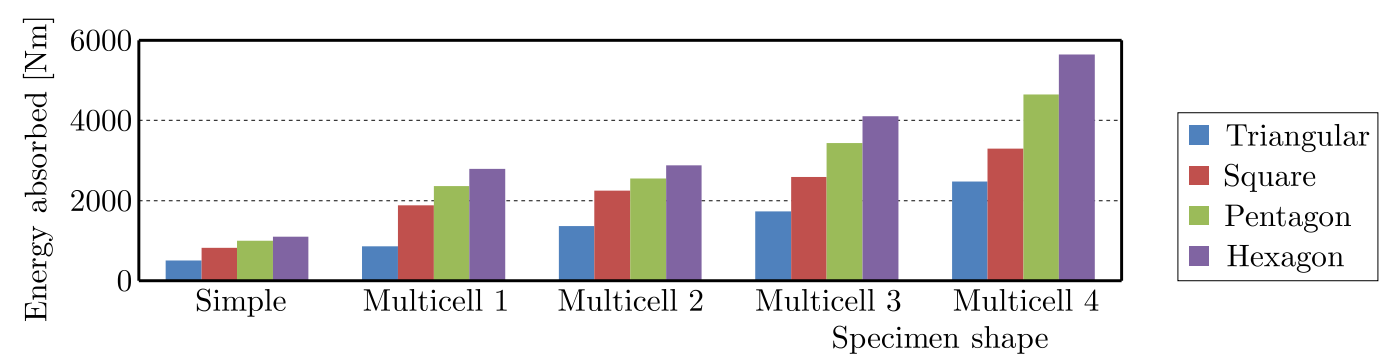

Fig. 4. Energy absorption capacity of various polygonal tubes

\subsection{Effective Stroke Ratio (ESR)}

The ESR values of triangular, square, pentagon and hexagon tubular sections are evaluated from Eq. (3.1), and the results are presented. The rank of polygonal tubes under axial compression is hexagonal, pentagonal, square and triangular. For the stainless steel specimens, hexagonal tubes have the most effective stroke length, and the number of folding elements in triangular tubes offers the least, see Fig. 5. Further, it is seen that the ESRs of various tubes mostly distribute in the range between 0.74 to 0.92 .
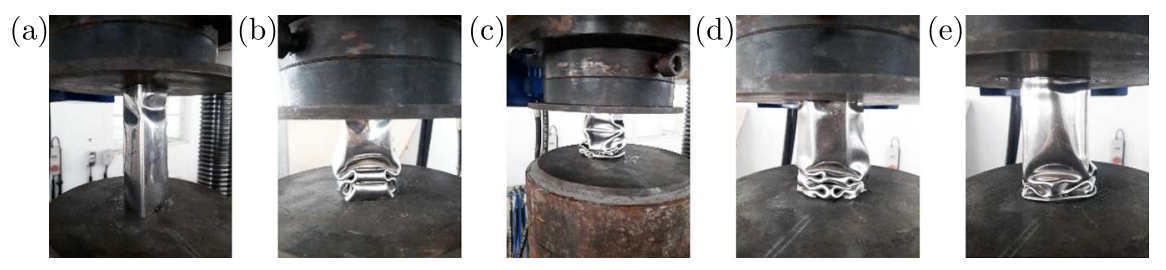

Fig. 5. Folding pattern of tested samples

\subsection{Specific Energy Absorption (SEA)}

The specific energy absorption defined by Eq. (3.2) is calculated and the results for all polygonal tubes are depicted in Fig. 6. It is noted that a hexagonal tube has the best specific energy absorption followed by a pentagonal, square and the triangular tube. The SEA the number and sides of the polygonal section have the highest energy absorption.

\subsection{Effectiveness of Energy Absorption (EEA)}

The effectiveness of energy absorption defined by Eq. (3.3) is calculated and the results for all polygonal tubes are depicted in Fig. 7. It is identified that the hexagonal tube has the best 


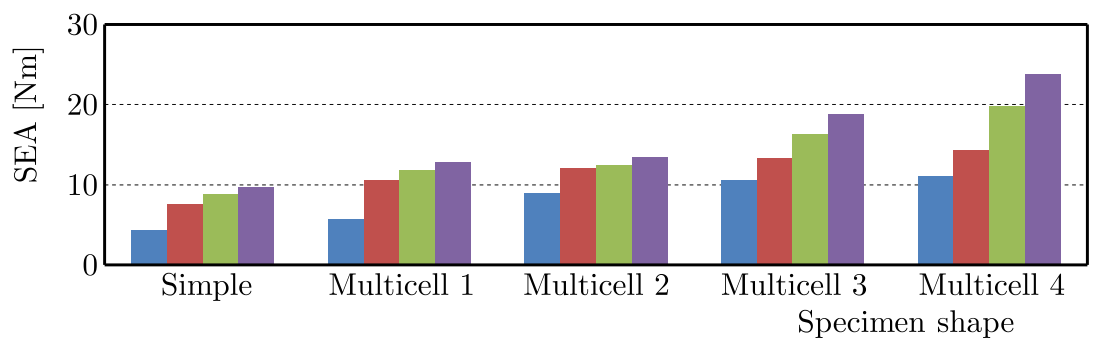

Triangular

- Square

Pentagon

- Hexagon

Fig. 6. Variation of SEAs with various polygonal tubes

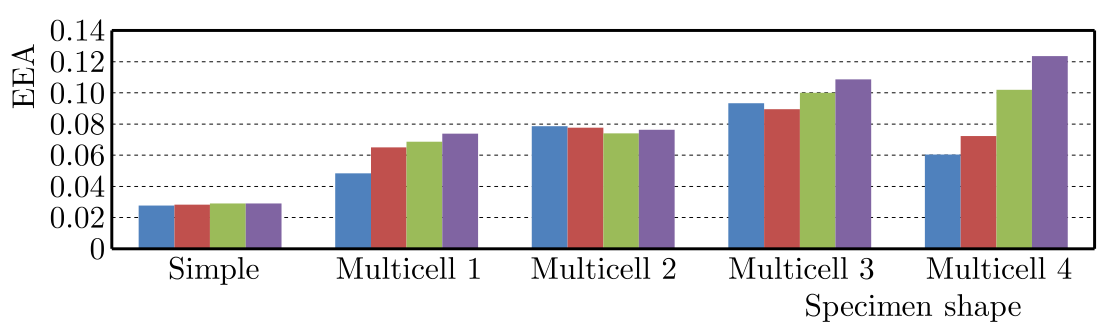

- Triangular

- Square

- Pentagon

Hexagon

Fig. 7. Variation of EEAs with various polygonal tubes

effectiveness of energy absorption followed by a triangular, square and the pentagonal tube. The EEA for a greater number of sides of the polygonal section has the highest energy absorption.

\subsection{Non-dimensional Load-carrying Capacity (NLC)}

The non-dimensional load-carrying capacity defined in Eq. (3.4) is calculated and the results for all polygonal sections are depicted in Fig. 8. As seen in Fig. 8, a reduction of NLC has occurred with an increase of the solidity ratio, and it is evident that the NLCs of hexagonal tubes are the best compared to all.

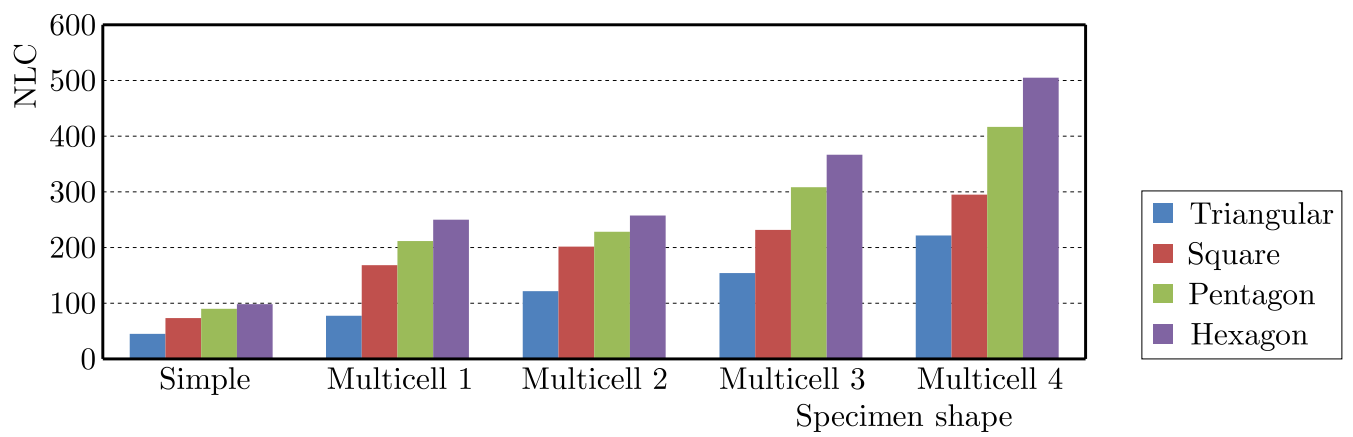

Fig. 8. Variation of NLCs with various polygonal tubes

Moreover, the deformation mode of hexagonal tubes is more stable than its NLC which is the strongestm and the deformation mode of triangular tubes is so unstable that its NLC is the weakest. With respect to the NLC, the rank of polygonal tubes under axial compression is (strongest to weakest) hexagonal, pentagonal, square and triangular. It is also noted that the NLCs of polygonal tubes could be improved with the increase of the number of sides of polygonal tubes. The number of sides has a great effect on the NLC.

\subsection{Stability of Load-carrying Capacity (SLC)}

The stability of load-carrying capacity defined by Eq. (3.5) is calculated and the results for all polygonal tubes are depicted in Fig. 9. It is seen that SLCs of all polygonal tubes increase with 
the solidity ratio. It is evident that hexagonal multi-cell tubes exhibit better stability of load-carrying capacity than that of pentagonal, square and triangular tubes with the same solidity ratio.
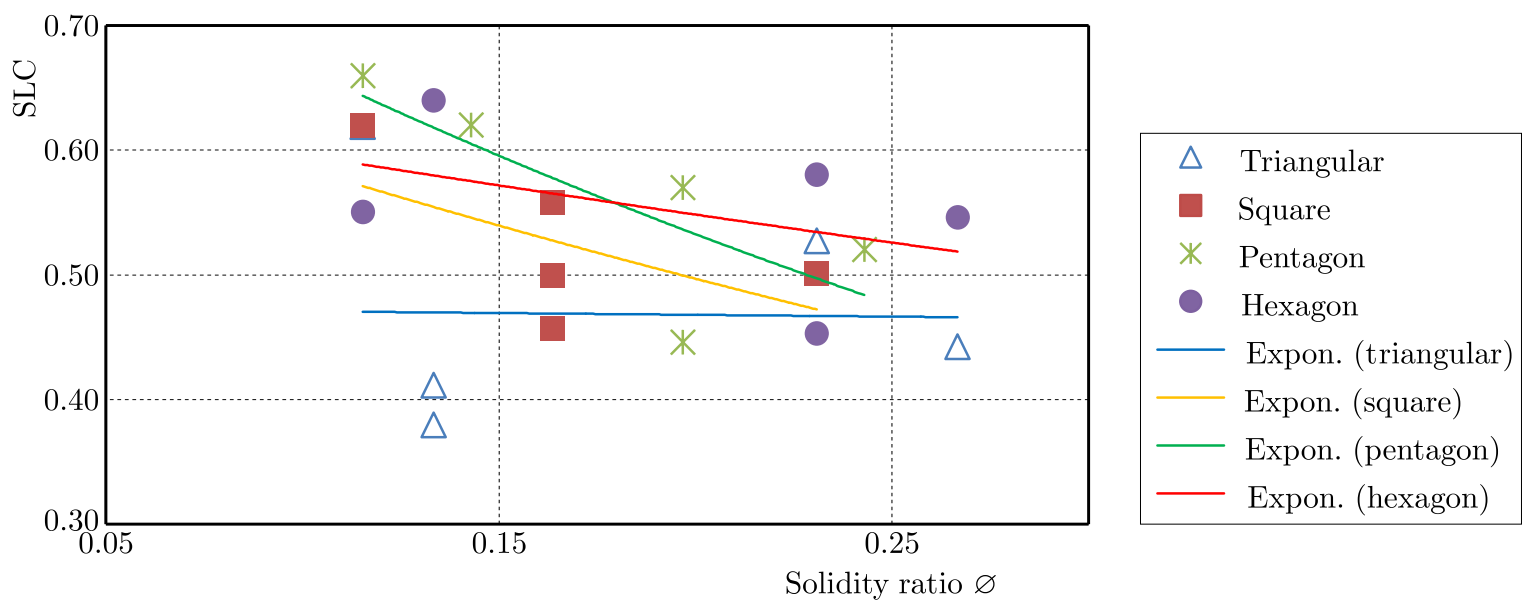

Fig. 9. Variation of SLCs with the solidity ratio of various polygonal tubes

\section{Numerical analysis}

Numerical simulations for axial compression of thin-walled structures are carried out using LS-DYNA software. A three-dimensional geometry model of the specimens was created in CATIA software and imported in LS-DYNA finite element analysis software. The model geometry consists of a thin-walled tube between two rigid parts as its ends. The dimensions of specimens were considered the same as in experiments, and wall thickness was $1.5 \mathrm{~mm}$, respectively, to ensure the same amount of material to be used. The same value of tube height $h=120 \mathrm{~mm}$ was applied for all specimens. The boundary conditions were the same as in the experimental tests. The upper end of the column was constrained rigidly, while the other end crashed with a constant velocity of $10 \mathrm{~mm} / \mathrm{min}$. Material properties and the Johnson-Cook strain rate parameters used in the simulation were $d_{1}=0.69, d_{2}=d_{3}=d_{5}=0, d_{4}=0.0546$. The tube was meshed in global contact by 4-node linear brick solid elements with reduced integration. Additionally, the material models for the tube and the rigid parts were Mat_picewise_linear_plasticity. The rigid ones were applied uniformly to all elements to balance computational efficiency and accuracy. In order to obtain deformations, the automatic surface-to-surface contact was used. The FE model of crashed sample patterns is shown in Fig. 10. The simulation results of energy absorption capacity are listed in Table 3 .

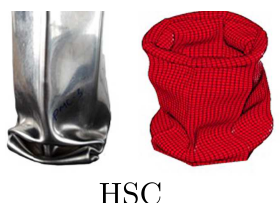

HSC

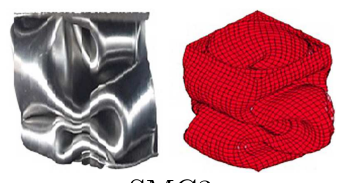

SMC3

Fig. 10. Sample crashed pattern of the FE model

\subsection{Numerical results}

The deformations of all thin-walled structures deform stably and progressively during the crashing process. The quantity of folds from a less to a greayer is trailed by triangular, square, pentagon and hexagon tubes. A progressively glowing number of folds implies increasing amount 
of material of the segment retaining a higher energy absorption efficiency. The crashing force rises up sharply and then falls down before the mean values soar up when the deformation capacity is exhausted at the effective stroke length. The load vs displacement curve obtained from this numerical model was similar to that obtained from the experiment. The crush force efficiency is an important factor to characterize the loading consistency. The specimens with a higher value of CFE is better for the load consistence. But the mean crashing force for some patterns is lower by $10 \%$, and the reason for that is good bonding not considered in the analysis. However, a circular hexagonal multi-cell column HMC4 is the overall best energy absorber in all other multi-cell types. The specific energy absorption of HMC4 is $20 \%$ and $27 \%$ higher than that of PMC4 and HMC3. CFE of HMC4 is increased by $4.5 \%$ and $7.8 \%$ over PMC4 and HMC3, respectively.

\subsection{Comparison of numerical and experimental results}

The average value of specific energy absorption for single and multi-cell tubular sections under quasi-static experimental results and FE results are tabulated, and a comparison of SEA is shown in Fig. 11. The influence of the number of sides in polygonal sections and the number of cells in specific energy absorption were studied. The variation of results between experimental and numerical was about $10 \%$ due to good bonding and computational accuracy. Experimental results were good in agreement with numerical analysis. From the tabulated results and graphs, the multi-cell sections show a higher rate of energy absorption than the simple cells. The value of SEA for HMC4 (SEA $23.3 \mathrm{kN}$ ) is greater than for the other sections, and the percentage of improvement is about $244 \%$ when compared to simple sections.

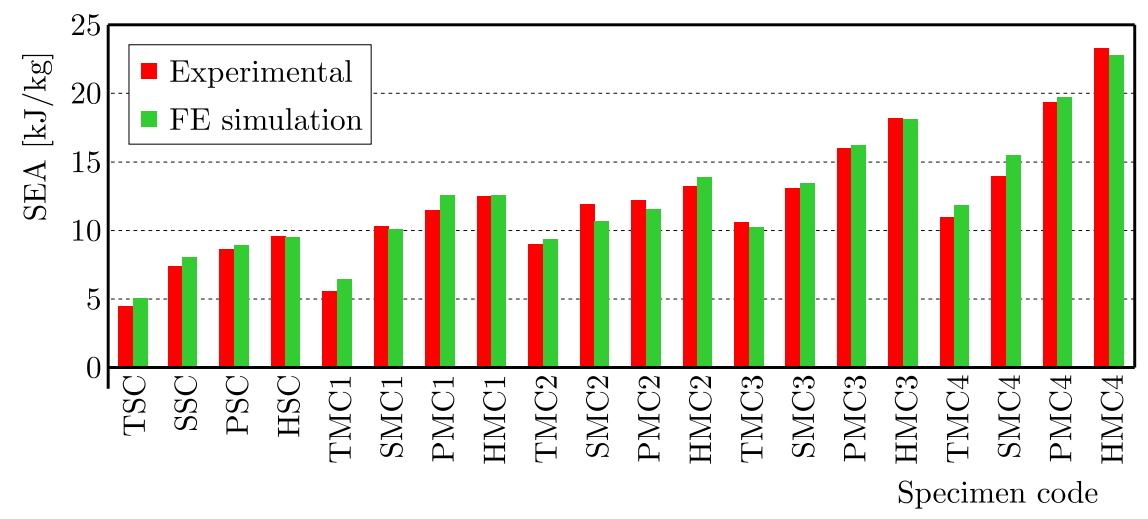

Fig. 11. Comparison of SEA results

\subsection{Parametric study}

A parametric study has been conducted to investigate the influence of geometrical parameters on crashworthiness. HMC4 specimen has been investigated in the parametric study because HMC4 is superior to all specimens in terms of specific energy absorption and crush force efficiency. In this investigation, different values of specimen length of $h=100 \mathrm{~mm}, h=120 \mathrm{~mm}$, $h=140 \mathrm{~mm}$ and different striking velocity of $5 \mathrm{~m} / \mathrm{s}, 10 \mathrm{~m} / \mathrm{s}$ and $15 \mathrm{~m} / \mathrm{s}$ have been considered.

\subsubsection{Effect of variation in length}

In this study, LS-DYNA was used to simulate the performance of a thin-walled tube under axial crashing. The variation in energy absorption was observed by varying the length of the specimen and crashing up to $60 \mathrm{~mm}$. Initially, the test was conducted with original dimensions, later the height of the specimen was altered by $20 \mathrm{~mm}$ addition and a $20 \mathrm{~mm}$ reduction in the original value. The load vs displacement of the test are tabulated in Table. 4. 
Table 3. Comparison of numerical results with experimental ones

\begin{tabular}{|c|c|c|c|c|c|c|c|c|c|c|}
\hline \multirow{2}{*}{ Code } & \multicolumn{2}{|c|}{$F_{\text {mean }}[\mathrm{kN}]$} & \multicolumn{2}{|c|}{$F_{\text {peak }}[\mathrm{kN}]$} & \multicolumn{2}{c|}{ TEA $[\mathrm{Nm}]$} & \multicolumn{2}{c|}{ SEA $[\mathrm{kJ} / \mathrm{kg}]$} & \multicolumn{2}{c|}{ CFE [\%] } \\
\cline { 2 - 12 } & Exp. & Num. & Exp. & Num. & Exp. & Num. & Exp. & Num. & Exp. & Num. \\
\hline \hline TSC & 6.33 & 7.10 & 16.67 & 17.91 & 506.4 & 568.0 & 4.44 & 4.98 & 38.0 & 39.6 \\
\hline SSC & 10.24 & 11.28 & 22.39 & 25.69 & 819.2 & 902.4 & 7.31 & 8.06 & 45.7 & 43.9 \\
\hline PSC & 12.52 & 12.93 & 28.07 & 25.30 & 1001.6 & 1034.4 & 8.63 & 8.92 & 44.6 & 51.1 \\
\hline HSC & 13.69 & 13.75 & 30.22 & 28.40 & 1095.2 & 1100.0 & 9.52 & 9.57 & 45.3 & 48.4 \\
\hline TMC1 & 10.81 & 12.54 & 26.27 & 30.27 & 864.8 & 1003.2 & 5.54 & 6.43 & 41.1 & 41.4 \\
\hline SMC1 & 23.54 & 23.12 & 47.13 & 45.50 & 1883.2 & 1849.6 & 10.35 & 10.16 & 49.9 & 50.8 \\
\hline PMC1 & 29.56 & 32.47 & 51.86 & 49.90 & 2364.8 & 2597.6 & 11.48 & 12.61 & 57.0 & 65.1 \\
\hline HMC1 & 34.86 & 35.25 & 60.07 & 58.03 & 2788.8 & 2820.0 & 12.45 & 12.59 & 58.0 & 60.7 \\
\hline TMC2 & 17.03 & 17.91 & 38.52 & 37.52 & 1362.4 & 1432.8 & 8.96 & 9.43 & 44.2 & 47.7 \\
\hline SMC2 & 28.12 & 25.43 & 56.13 & 51.02 & 2249.6 & 2034.4 & 11.84 & 10.71 & 50.1 & 49.8 \\
\hline PMC2 & 31.85 & 30.65 & 61.21 & 56.36 & 2548 & 2452.0 & 12.13 & 11.68 & 52.0 & 54.4 \\
\hline HMC2 & 36.01 & 37.89 & 65.94 & 64.06 & 2880.8 & 3031.2 & 13.21 & 13.90 & 54.6 & 59.1 \\
\hline TMC3 & 21.56 & 21.00 & 40.91 & 40.24 & 1724.8 & 1680.0 & 10.52 & 10.24 & 52.7 & 52.2 \\
\hline SMC3 & 32.38 & 33.39 & 58.08 & 58.90 & 2590.4 & 2671.2 & 13.08 & 13.49 & 55.8 & 56.7 \\
\hline PMC3 & 43.01 & 43.44 & 69.37 & 67.80 & 3440.8 & 3475.2 & 16.08 & 16.29 & 62.0 & 64.1 \\
\hline HMC3 & 51.26 & 51.21 & 80.12 & 83.20 & 4100.8 & 4096.8 & 18.31 & 18.30 & 64.0 & 61.6 \\
\hline TMC4 & 30.99 & 33.85 & 50.08 & 49.86 & 2479.2 & 2708.0 & 10.97 & 11.98 & 61.9 & 67.9 \\
\hline SMC4 & 41.24 & 45.90 & 66.50 & 68.19 & 3299.2 & 3672.0 & 13.98 & 15.56 & 62.0 & 67.7 \\
\hline PMC4 & 58.13 & 59.48 & 88.07 & 88.25 & 4650.4 & 4758.4 & 19.38 & 19.83 & 66.0 & 67.4 \\
\hline HMC4 & 70.51 & 69.61 & 102.18 & 100.90 & 5640.8 & 5568.8 & 23.31 & 23.01 & 69.0 & 69.0 \\
\hline
\end{tabular}

Table 4. Effect of variation in length on energy absorption (EA)

\begin{tabular}{|c|c|c|c|c|c|}
\hline $\begin{array}{c}\text { Height of } \\
\text { specimen }[\mathrm{mm}]\end{array}$ & $\begin{array}{c}F_{\text {mean }} \\
{[\mathrm{kN}]}\end{array}$ & $\begin{array}{c}F_{\text {peak }} \\
{[\mathrm{kN}]}\end{array}$ & $\begin{array}{c}\mathrm{EA} \\
{[\mathrm{J} / \mathrm{kg}]}\end{array}$ & $\begin{array}{c}\text { SEA } \\
{[\mathrm{J} / \mathrm{kg}]}\end{array}$ & $\begin{array}{c}\mathrm{CFE} \\
{[\%]}\end{array}$ \\
\hline \hline 100 & 56.91 & 82.23 & 4552.8 & 20325.0 & 69.2 \\
\hline 120 & 58.28 & 87.34 & 4662.4 & 19266.1 & 66.7 \\
\hline 140 & 52.9 & 78.8 & 4232.0 & 16276.9 & 67.1 \\
\hline
\end{tabular}

\subsection{Effect of striking velocity}

The striking velocity was also modified to investigate the variation of energy absorption of the specimen. Three different crashing velocities were applied 5, 10 and $15 \mathrm{~mm} / \mathrm{min}$. An attempt was made to study the effect of various crashing speeds on the specimen. The observations made are represented as load vs displacement test results tabulated in Table 5.

Table 5. Effect of variation in velocity on energy absorption

\begin{tabular}{|c|c|c|c|c|c|}
\hline $\begin{array}{c}\text { Striking velocity } \\
{[\mathrm{mm} / \mathrm{min}]}\end{array}$ & $\begin{array}{c}F_{\text {mean }} \\
{[\mathrm{kN}]}\end{array}$ & $\begin{array}{c}F_{\text {peak }} \\
{[\mathrm{kN}]}\end{array}$ & $\begin{array}{c}\text { EA } \\
{[\mathrm{J} / \mathrm{kg}]}\end{array}$ & $\begin{array}{c}\text { SEA } \\
{[\mathrm{J} / \mathrm{kg}]}\end{array}$ & $\begin{array}{c}\text { CFE } \\
{[\%]}\end{array}$ \\
\hline \hline 5 & 59.4 & 88.1 & 4752.0 & 19636.4 & 67.5 \\
\hline 10 & 69.61 & 100.9 & 5568.8 & 23011.6 & 69.0 \\
\hline 15 & 73.45 & 104.3 & 5876.0 & 24281.0 & 70.4 \\
\hline
\end{tabular}




\section{Conclusion}

In this research, a set of non-dimensional Key Performing Indicators (KPIs) and Specific Energy Absorption (SEA) have been proposed to evaluate the performance and behaviour of tubular structures that are used energy absorbers. By utilizing the KPIs, throughout analysis and comparison of the energy performance of single tubes and multi-cell tubes have been conducted. It has been predicted from those results that the values of the SEA, NLC and EEA can be improved with the increase in the number of sides of polygonal tubes. The circular tube (multi-cell 4) possesses the best energy absorption performance, superior to that of all remaining tubes. The evaluations of KPIs for polygonal tubes under axial loading reveal that the energy absorption capacity of circular hexagonal tubes is greater than that of triangular, square and pentagonal tubes. Their KPIs as functions of the solidity ratio usually follow the similar trends.

Based on experiments and simulations, the absorbed energy per unit mass is maximum for circular hexagonal tubes. A parametric study of circular hexagonal tubes has been carried out. It is found that the gradual increase in length of the samples affects their energy absorption capacity, lowering the striking velocity. So, cylindrical multi-cell thin-walled columns are superior to conventional structures as energy absorbers. The energy absorption of multi-cell columns is improved by adopting some cylindrical shell as webs. The KPIs can be used to design and development of thin-walled structures with a certain level of optimization.

\section{References}

1. Abramowicz W., Jones N., 1984, Dynamic axial crashing of circular tubes, International Journal of Impact Engineering, 2, 3, 263-281

2. Abramowicz W., Jones N., 1986, Dynamic progressive buckling of circular and square tubes, International Journal of Impact Engineering, 4, 4, 243-270

3. Baroutaji A., SajJia M., Olabi A.G., 2017, On the crashworthiness performance of thin-walled energy absorbers: recent advances and future developments, Thin-Walled Structures, 118, 137-163

4. Bigdeli A., Nouri M.D., 2019, A crushing analysis and multi-objective optimization of thin-walled five-cell structures, Thin-Walled Structures, 137, 1-18

5. Deng X., LiU W., 2019, Multi-objective optimization of thin-walled sandwich tubes with lateral corrugated tubes in the middle for energy absorption, Thin-Walled Structures, 137, 303-317

6. Hu L.L., He X.L., Wu G.P., Yu T.X., 2015, Dynamic crushing of the circular-celled honeycombs under out-of-plane impact, International Journal of Impact Engineering, 75, 150-161

7. Hussein R.D., Ruan D., Lu G., Guillow S., Yoon J.W., 2017, Crushing response of square aluminium tubes filled with polyurethane foam and aluminium honeycomb, Thin-Walled Structures, 110, 140-154

8. Li Z., Chen R., Lu F., 2018, Comparative analysis of crashworthiness of empty and foam-filled thin-walled tubes, Thin-Walled Structures, 124, 343-349

9. Markiewicz E., Ducroce P., Drazetic P., 1998, An inverse approach to determine the constitutive model parameters from axial crushing of thin-walled square tubes, International Journal of Impact Engineering, 21, 6, 433-449

10. Mozafari H., Khatami S., Molatefi H., Crupi V., Epasto G., Guglielmino E., 2016, Finite element analysis of foam-filled honeycomb structures under impact loading and crashworthiness design, International Journal of Crashworthiness, 21, 2, 148-160

11. Nia A.A., Parsapour M., 2014, Comparative analysis of energy absorption capacity of simple and multi-cell thin-walled tubes with triangular, square, hexagonal and octagonal sections, Thin-Walled Structures, 74, 155-165 
12. Olabi A.G., Morris E., Hashmi M.S.J., 2007, Metallic tube type energy absorbers: a synopsis, Thin-Walled Structures, 45, 7-8, 706-726

13. Pyrz M., Krzywoblocki M., 2017, Crashworthiness optimization of thin-walled tubes using Macro Element Method and Evolutionary Algorithm, Thin-Walled Structures, 112, 12-19

14. Sharifi S., Shakeri M., Fakhari H.E., Bodaghi M., 2015, Experimental investigation of bitubal circular energy absorbers under quasi-static axial load, Thin-Walled Structures, 89, 42-53

15. Sun G., Deng M., Zheng G., Li Q., 2019, Design for cost performance of crashworthy structures made of high strength steel, Thin-Walled Structures, 138, 458-472

16. Sun G., Liu T., Huang X., Zheng G., Li Q., 2018, Topological configuration analysis and design for foam filled multi-cell tubes, Engineering Structures, 155, 235-250

17. Sun G., PAng T., FAng J., Li G., Li Q., 2017, Parameterization of criss-cross configurations for multiobjective crashworthiness optimization, International Journal of Mechanical Sciences, 124, 145-157

18. Umeda T., Mimura K., Morisaka T., 2010, Study of energy absorption efficiency for a few thin-walled tubes in axial crushing, Journal of Solid Mechanics and Materials Engineering, 4, 7, 875-890

19. Vinayagar K., Kumar A.S., 2017a, Crashworthiness analysis of double section bi-tubular thin-walled structures, Thin-Walled Structures, 112, 184-193

20. Vinayagar K., Kumar A.S., 2017b, Multi-response optimization of crashworthiness parameters of bi-tubular structures, Steel and Composite Structures, 23, 1, 31-40

21. Wu S., Zheng G., Sun G., Liu Q., Li G., Li Q., 2016. On design of multi-cell thin-wall structures for crashworthiness, International Journal of Impact Engineering, 88, 102-117

22. Yin H., Wen G., Liu Z., QIng Q., 2014, Crashworthiness optimization design for foam-filled multi-cell thin-walled structures, Thin-Walled Structures, 75, 8-17

23. Yu T.X., Xiang Y.F., Wang M., Yang L.M., 2014, Key performance indicators of tubes used as energy absorbers, Key Engineering Materials, 626, 155-161

24. Zhang Y., Ge P., Lu M., Lai X., 2018a, Crashworthiness study for multi-cell composite filling structures, International Journal of Crashworthiness, 23, 1, 32-46

25. Zhang Y., Xu X., Wang J., Chen T., Wang C.H., 2018b, Crushing analysis for novel bio-inspired hierarchical circular structures subjected to axial load, International Journal of Mechanical Sciences, 140, 407-431 